\title{
Strengthening encoding via implementation intention formation increases prospective memory commission errors
}

\author{
Julie M. Bugg • Michael K. Scullin • Mark A. McDaniel
}

Published online: 25 January 2013

(C) Psychonomic Society, Inc. 2013

\begin{abstract}
Prospective memory refers to the ability to remember to execute future intentions (e.g., taking medication with dinner). Although most prior research on prospective memory errors has focused on omission errors (i.e., failures to perform an intention in response to a target cue), there has been a recent surge in research on commission errors, the erroneous performance of a finished intention. Existing studies have examined factors at retrieval that lead to commission errors; the present study extends this research by investigating the impact of encoding strength. We found that relative to standard encoding, implementation intention encoding doubled the risk of commission errors in our laboratory paradigm for both young and older adults. This novel finding demonstrates the impact of encoding strength on commission errors and documents the potential challenges of deactivating the effects of implementation intentions upon completion of a prospective memory task.
\end{abstract}

Keywords Prospective memory $\cdot$ Commission errors · Implementation intentions $\cdot$ Finished intentions $\cdot$ Aging

The systematic study of retrospective memory errors (e.g., false memories), though a surprisingly late undertaking (Roediger, 1996a), has been influential in theorizing about memory. Retrospective memory researchers have demonstrated the importance of qualitatively different types of errors in revealing how memory operates. Prospective memory (PM) researchers have followed suit by broadening their investigation of PM errors. PM refers to the ability to remember to execute future

J. M. Bugg $(\bowtie) \cdot$ M. K. Scullin $\cdot$ M. A. McDaniel

Department of Psychology, Washington University in St. Louis,

St. Louis, MO 63130-4899, USA

e-mail: jbugg@artsci.wustl.edu

M. K. Scullin

Emory University School of Medicine, Atlanta, GA, USA intentions in response to a target cue. For example, one might need to remember to take a daily medication, and seeing the medication bottle may cue the intention. Omission errors (e.g., failure to take the medication) have been documented since the initial studies of PM, but until very recently, little attention has been paid to errors of the commission type. These refer to errors in which participants erroneously perform a finished intention (e.g., taking a second dose of medication upon encountering the bottle later that day).

In a typical PM paradigm, participants are instructed to make a response (e.g., to press F1) whenever they encounter a target cue (e.g., the word "horse"), which occurs very infrequently during an ongoing task (e.g., lexical decision). Using a novel extension of this paradigm (cf. Scullin, Bugg, McDaniel, \& Einstein, 2011; Scullin, Einstein, \& McDaniel, 2009), Scullin, Bugg and McDaniel (2012) revealed commission errors, the first of their kind in a nonhabitual-PM paradigm (for repetition errors in a habitual-PM task, see Einstein, McDaniel, Smith, \& Shaw, 1998). As is illustrated in Fig. 1, following an active-PM phase (i.e., responding to target cues during lexical decision), participants engaged in a finished-PM phase, which also included lexical decision. Importantly, prior to the finished-PM phase, the participants had been instructed that the PM task was finished and that they would no longer need to press the designated key in response to target cues. The target cues continued to be presented during the finished-PM phase, and commission errors were observed (see Pink \& Dodson, 2012; Walser, Fischer, \& Goschke, 2012, for similar results).

\section{Factors influencing commission errors}

Prospective remembering shares several similarities with retrospective remembering (see, e.g., Roediger, 1996b), and therefore understanding PM commission errors is likely to be facilitated by examining the extant retrospective 
Fig. 1 Illustration of the commission-error paradigm. $\mathrm{PM}=$ prospective memory

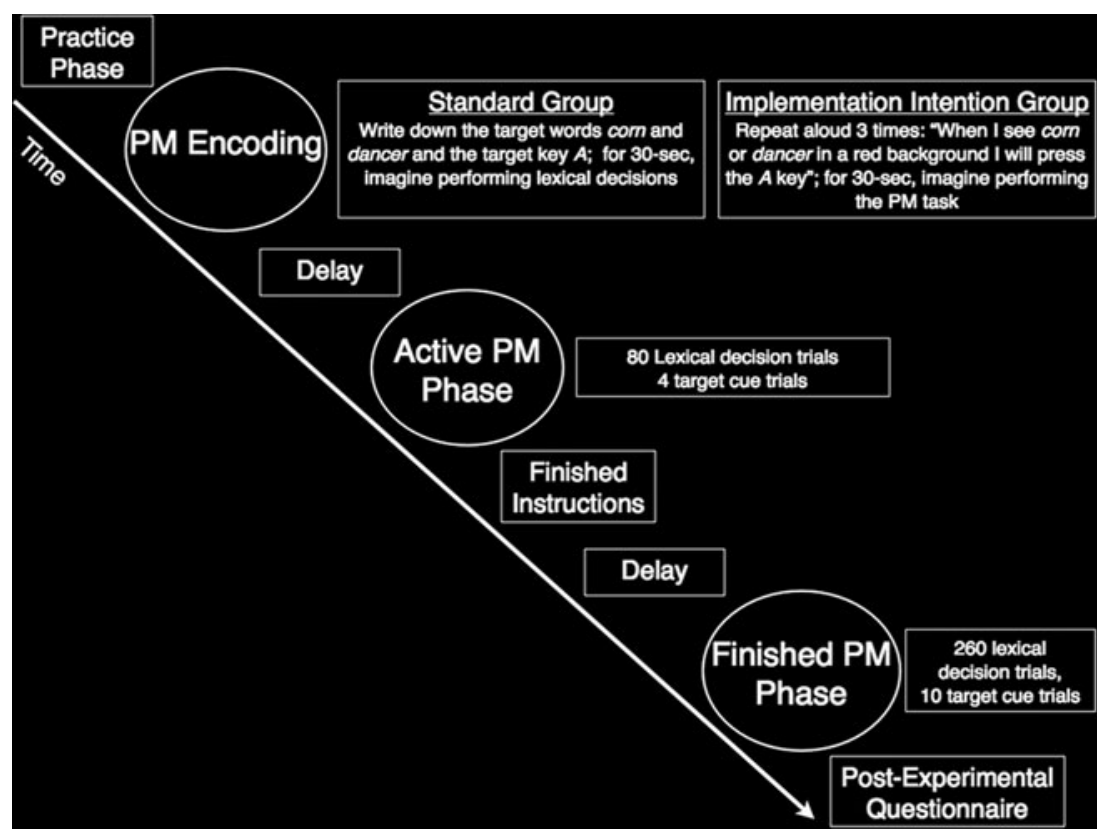

memory commission-error literature. A classic example is false recall of words that were related to studied words but that were not presented (e.g., Deese, 1959; Roediger \& McDermott, 1995). Early studies pointed to the strength of backward associations - the likelihood that a critical lure would spontaneously come to mind when items were encoded during study-in modulating the level of false memories (Deese, 1959). By contrast, later theories placed more emphasis on retrieval processes such as source monitoring (e.g., Johnson, Hashtroudi, \& Lindsay, 1993) and misattributions of fluency (i.e., Jacoby, Kelley, \& Dywan, 1989).

A parallel discussion regarding the contribution of encoding and retrieval processes is emerging in investigations of PM commission errors, although the initial focus has been on retrieval processes. Walser et al. (2012) found that commission errors were more likely when participants were strategically monitoring for a new, but similar, PM intention, and Pink and Dodson (2012) showed that dividing attention increased commission errors for cues that were habitually responded to in a prior phase. Moreover, Scullin and Bugg (2012) found that the risk of commission errors increased for participants who were fatigued during the finished-PM phase. In these studies, the interpretation was that the initial encodings of the PM intention were equivalent across groups, and that factors occurring during the retrieval phase (i.e., the finished-PM phase) determined commission-error risk.

The effects of encoding on PM commission errors have not previously been isolated. Two studies have shown that the likelihood of commission errors is influenced by the contextual overlap between the active-PM and finished-PM phases (Scullin et al., 2012; cf. Walser et al., 2012), consistent with interactions taking place between encoding and retrieval (i.e., transfer-appropriate processing). Commission errors were highest when the cue was salient (e.g., placed on a distinctively colored background screen) and the ongoing task was matched across phases, while zero young or older participants produced a commission error when contextual overlap was very low (Scullin et al., 2011).

In the present study, we aimed to examine the influence of encoding on PM commission errors. The key question was whether the initial strategy used to encode an intention has any bearing on the likelihood of a later commission error. Applying Deese's (1959) work on backward association strength (cf. Roediger, Watson, McDermott, \& Gallo, 2001) to the PM commission-error paradigm, an effect of encoding would be expected to the extent that it affected the likelihood that the no-longer-relevant intention would spontaneously come to mind when target cues were encountered in the finished-PM phase. We chose to manipulate the strength of encoding by comparing implementation intention (II) encoding (Gollwitzer, 1999), which forges strong cue-intention associative links, to standard encoding.

\section{II encoding}

II encoding is a simple strategy that benefits a wide range of PM tasks. The strategy consists of a statement of the form "If situation $x$ occurs, then I will perform intention $y$," and it is often accompanied by mental visualization of the intention execution (Gollwitzer, 1999). II encoding has been demonstrated to reduce forgetting in a variety of daily tasks (e.g., adherence to blood glucose monitoring; Liu \& Park, 2004). It has also been shown to improve PM performance in some laboratory paradigms (Cohen \& Gollwitzer, 2008; McDaniel, Howard, \& Butler, 2008; McDaniel \& Scullin, 
2010). A critical question regarding II encoding that has been raised (Marsh, Hicks, Cook, \& Mayhorn, 2007), but never tested, is whether there is potentially a deleterious consequence associated with this encoding strategy (cf. Meiser \& Rummel, 2012, who showed increased false alarms during an active-PM task using an II-like encoding strategy). We propose that the robust cue-intention link forged by II (e.g., Gollwitzer, 1999; McFarland \& Glisky, 2012), which has been hypothesized to produce automatic intention execution (Gollwitzer, 1999) or spontaneous intention retrieval (McDaniel \& Scullin, 2010), may exacerbate the difficulty of "turning off" a completed intention and increase commission-error risk. Indirect support for this possibility has stemmed from findings of greater intention interference (i.e., slowed responding to a PM cue; Cohen, Dixon, \& Lindsay, 2005) or commission errors when previously relevant cues appeared immediately after a PM task was finished, presumably at a point when the cue-action link was still quite robust (Walser et al., 2012; West, McNerney, \& Travers, 2007).

Our primary interest was in examining whether II encoding might increase commission errors relative to standard encoding. We used II encoding instructions that were identical to those in previous work (including the same target cues) that had shown that II reliably improved PM performance relative to standard instructions (McDaniel \& Scullin, 2010). However, for the present purposes, enhanced PM hits during the active-PM phase in the II condition could cloud interpretation of the effects of encoding on commission errors in the finished-PM phase. Accordingly, to avoid this possible contaminating factor occurring during the active-PM phase (Marsh et al., 2007), we used salient PM cues to ensure high levels of PM hits across encoding conditions (Scullin et al., 2012). Thus, the advantage of the present paradigm was that we adopted II instructions that are known to be potent with the very materials used herein (McDaniel \& Scullin, 2010; see also McDaniel et al., 2008), but we negated the potential contaminating effects of differential PM responding in the active-PM phase on the effects of interest in the finished-PM phase.

A second goal was to examine whether II encoding might also increase commission errors for older adults. Several studies have focused on II encoding within an older adult sample (e.g., Chasteen, Park, \& Schwarz, 2001). Some evidence has suggested that the spontaneous retrieval processes supporting PM (processes presumably enhanced by implementation intentions; McDaniel \& Scullin, 2010), unlike controlled retrieval processes (McDaniel, Einstein, \& Jacoby, 2008), are relatively preserved in normal aging (McDaniel \& Einstein, 2007). Accordingly, we predicted that commission errors would be significantly higher for participants in the II condition, regardless of age group.

\section{Method}

Participants and design

A group of 63 Washington University undergraduates $\left(M_{\text {age }}=\right.$ 19.75 years, $S D=1.40$; range $=18-25 ; 54 \%$ female $)$ and 47 community-dwelling older adults $\left(M_{\mathrm{age}}=71.38\right.$ years, $S D=$ 5.62 ; range $=60-85 ; 70 \%$ female) who reported normal or corrected vision and hearing participated.

We employed a $2 \times 2$ between-subjects factorial design that included the variables encoding strategy (II or standard) and age group (younger or older). Participants were randomly assigned to II (younger $n=31$, older $n=$ 24) and standard encoding conditions (younger $n=32$, older $n=23$ ).

\section{Materials}

The materials were identical to those employed by Scullin et al. (2012) (cf. McDaniel \& Scullin, 2010). The target words (corn/dancer or fish/writer) always appeared in white font against salient red or blue background screens (target pairs and background colors were counterbalanced across participants). All other words were presented in white font against a black background.

\section{Procedure}

The procedure was identical to Scullin et al.'s (2012) salientcue/task-match condition, with the exception of the encoding manipulation (see Fig. 1). Participants first received instructions and practice with the ongoing lexical-decision task. They were instructed to respond as quickly and accurately as possible to whether a string of letters represented a word or nonword by pressing keys marked $Y$ and $N$ ( 5 and 6 on the number pad, respectively).

Participants next encoded the PM intention. They were told to press the $A$ key if they saw the target words corn or dancer (or, in counterbalance, fish/writer). They were further told that the target words would always appear in a red (or, in counterbalance, blue) background screen. In the standard encoding condition, participants were told to write down the two target words, and the target key (i.e., $A$ ). They then spent $30 \mathrm{~s}$ mentally imagining performing the lexicaldecision task. In the II encoding condition, participants had to say the following statement aloud three times: "When I see corn or dancer [fish or writer] in a red [blue] background, I will press the $A$ key." These participants then spent 30 s mentally imagining pressing the $A$ key when seeing the target words (e.g., Cohen \& Gollwitzer, 2008; McDaniel \& Scullin, 2010). All participants were given a ten-trial lexical-decision practice block that included two targets, and accuracy and speed feedback following each trial. 
To provide a delay between the encoding and test phases (Einstein \& McDaniel, 1990), participants took a vocabulary test and completed a demographics form, which took approximately $5 \mathrm{~min}$. Then they performed the active-PM phase (see Fig. 1), in which four of the 80 trials included a target word. As in previous work (Scullin et al., 2009, 2011, 2012), following this activePM phase, participants were instructed that they no longer needed to press the $A$ key in the presence of target words. They were further instructed that they would be performing more lexical-decision trials and that their only goal was to make the word/nonword decisions as quickly and accurately as possible. Prior to the finished-PM phase, we instituted a delay of approximately $5 \mathrm{~min}$, in which participants performed a 24-trial lexical-decision block and took a second vocabulary test. Then participants performed the finishedPM phase (see Fig. 1), in which ten of the 260 trials included a target word. Target words appeared in the same salient background screen as during the active-PM phase. Following the finished-PM phase, participants were asked to recall the target words and target key and were further queried as to whether they had ever pressed the $A$ key after being instructed to no longer do so (and if so, why).

\section{Results}

Prospective memory hits in the active-PM phase

Alpha was set to $p<.05$. We conducted $2 \times 2$ betweensubjects analyses of variance that included the encoding condition (II, standard) and age group (younger, older) variables for the proportions of $A$ presses to targets (PM hits). As expected, the proportion of PM hits was at ceiling $(M=.96)$. Neither the age group nor the encoding condition main effect was significant (both $F \mathrm{~s}<1$ ), nor was the Age Group $\times$ Encoding Condition interaction, $F(1,106)=$ 3.92, $M S E=.01, p<.10, \eta_{\mathrm{p}}^{2}=.04$ (standard: $M_{\text {young }}=$ $.95, M_{\text {older }}=.98 ;$ II: $\left.M_{\text {young }}=.98, M_{\text {older }}=.93\right)$.

Commission errors in the finished-PM phase

A commission error was defined as an $A$ press during the finished-PM phase (Scullin et al., 2012). The proportions of participants who made at least one commission error are presented in Fig. 2. Participants were significantly more likely to make a commission error following II encoding than following standard encoding, $\chi^{2}(1)=7.70, p<.01, \phi=.26$. This pattern was obtained for the younger adults, $\chi^{2}(1)=3.84, p<$ $.05, \phi=.25$, and for the older adults, $\chi^{2}(1)=3.85, p<.05, \phi=$ .29. Older adults demonstrated nominally more commission errors than did younger adults, but the age effect was not significant in the standard or II conditions (both $\chi^{2} \mathbf{s}<1$ ). ${ }^{1}$

\section{Discussion}

The primary finding was that II encoding doubled the risk for commission errors in younger and older adults in our laboratory paradigm. Theoretically speaking, the present finding provides novel evidence for the importance of encoding processes in modulating PM commission errors. This finding extends previous research that pointed to the importance of processes occurring at the time of intention retrieval (e.g., fatigue or divided attention) for modulating commission errors (Pink \& Dodson, 2012; Scullin \& Bugg, 2012; Scullin et al., 2012; Walser et al., 2012).

The significant increase in commission errors produced by II encoding suggests that a stronger encoding of the cueintention association may exacerbate the difficulty of deactivating a finished intention. Presumably, the association formed by II promoted spontaneous retrieval of the nolonger-relevant intention in the finished-PM phase ${ }^{2}$ (e.g., McDaniel \& Scullin, 2010). This finding stimulates the question of whether, following completion of an IIencoded intention, stronger deactivation of the intention (than is necessary for standard encoding) is needed. Future studies might examine whether the increased risk of commission errors associated with II encoding is minimized if participants create an II for deactivation purposes or replace the old action with a new action (but see Walser et al., 2012, for increased commission errors following encoding a new intention). ${ }^{3}$ Such studies would address the question of how readily one can "overwrite" the effects of II encoding.

One surprising finding was that we did not replicate the age-related (significant) increase in commission errors that

\footnotetext{
${ }^{1}$ An identical pattern was found when analyzing the total commission errors per participant.

${ }^{2}$ Subjectively, participants' reports coincided with the idea that retrieval was more automatic-like in the II condition, in which nine of 55 participants reported automatic-like commission errors (e.g., "pressing it was closer to a reflex," "I continued to press $A$ out of force of habit"), which was statistically greater than the one of 55 participants who reported such errors in the standard condition, $\chi^{2}(1)=7.04, p<.01, \phi=.25$. The two encoding conditions did not differ in the frequency of other types of selfreported errors (e.g., memory error/contextual confusion, "better safe than sorry," and output monitoring; see Scullin \& Bugg, 2012), $\chi^{2} \mathrm{~s}<1.09$. These self-report data should be viewed cautiously, as they are descriptive and not diagnostic; participants' reports were collected after they had learned that they should not have made commission errors. Examining total commission errors per participant indicated that participants who selfreported automatic-like commission errors $(M=6.80)$ did not commit more errors on average than did participants who self-reported other types of errors (e.g., memory error/contextual confusion $[M=8.47]$, better safe than sorry $[M=10.0]$, output monitoring $[M=7.56]), F(1,35)=1.41$, $M S E=9.30, p=.26$.

${ }^{3}$ We thank two anonymous reviewers for suggesting these ideas.
} 
Fig. 2 Proportions of participants who made at least one commission error as a function of encoding condition and age group. Error bars reflect standard errors

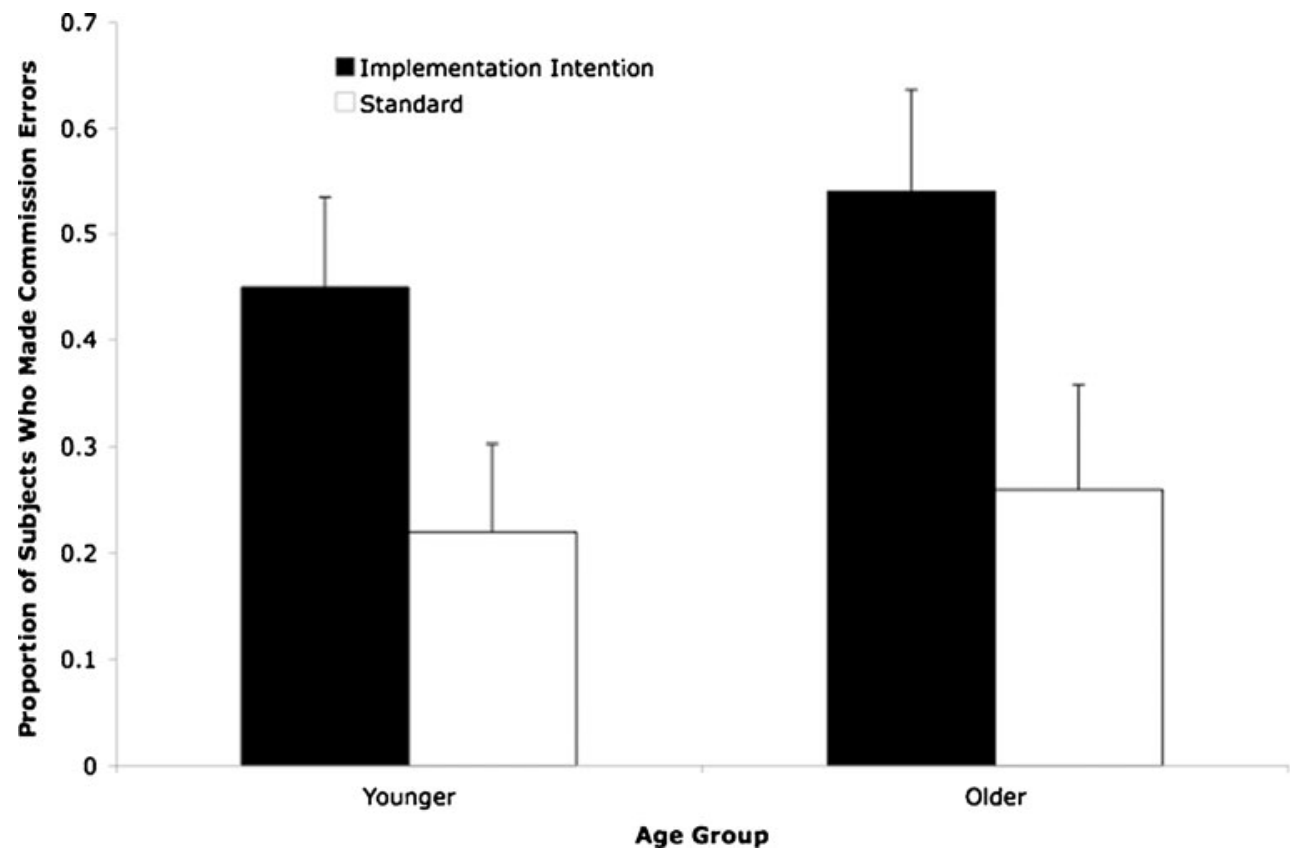

Scullin et al. (2012) observed using a standard encoding condition. At present, there are too few studies with older adults to ascertain whether we have failed to replicate a reliable finding. Our finding is in fact similar to that of Cohen et al. (2005), who found no significant age differences when comparing intention interference to no-longer-relevant cues between younger and older adults. Nevertheless, there are several possible explanations. One stems from a consideration of Scullin et al. (2012), who demonstrated that commission errors reflect not only spontaneous retrieval of the intention, but also the ability to inhibit the tendency to respond when the completed intention is retrieved. The present sample's executive control/inhibitory ability was better than that of the older adult sample tested by Scullin et al. (2012), which may explain the differential findings across standard encoding conditions. ${ }^{4}$ The absence of an age effect in the II encoding condition may reflect that preservation of spontaneous retrieval is possibly a stronger predictor of commission errors when II encoding is used (cf. Meiser \& Rummel, 2012). That is, executive/inhibitory control may account for less variance under conditions that produce more automatized responding, such as II (e.g., Gollwitzer, 1999).

\footnotetext{
${ }^{4}$ Following the PM phases, we gave older adults Stroop and Trail Making tests (see Scullin et al., 2012). Trail Making B performance was better in the present sample $(M=71.27 \mathrm{~s})$ than in the previous sample $(M=78.27 \mathrm{~s})$, though Stroop performance was similar between the studies $\left(M_{\text {curr }}=-32.26 ; M_{\text {prev }}=-32.97\right)$. In the present study, we found effects comparable to those in Scullin et al. (2012) in the standard encoding condition (worse Z-inhibit scores [reflecting a composite of Stroop and Trails B] for those who made commission errors [-.29] than those who did not [.18], but the effect was not significant, presumably due to low statistical power). Z-inhibit scores were nearly identical between those who made a commission error $(M=-.06)$ and those who did not $(M=-.05)$ in the II group.
}

Practically speaking, the novel finding of II encoding increasing commission-error risk in the laboratory confirmed an earlier conjecture (Marsh et al., 2007) that forming an II can have potentially unwanted consequences for both younger and older adults. Though we continue to advocate the many positive aspects of II for PM performance, the present findings suggest caution in employing II to achieve behaviors/actions that are only temporarily relevant or for which erroneous repetitions are harmful. In the domain of cardiovascular health, for example, a patient might formulate an II to remember to take an anticoagulant medication on Mondays, Wednesdays, and Fridays; however, because physicians often adjust the frequency and dosage of anticoagulants in response to fluctuations in blood thinness, the patient may be asked to take it only on Wednesdays. Indeed, the clinical significance of commission errors in anticoagulation control has been documented (Kimmel et al., 2007).

In summary, how one initially encodes an intention does influence the risk of PM commission errors. Future research might therefore address whether (and how) II encoding might be modified to promote effective intention completion without increasing commission-error risk. Borrowing once more from the false-memory literature, an exciting possibility is that a simple warning accompanying II (i.e., about the likelihood of commission errors) might reduce commission errors in PM. Warnings that precede encoding (e.g., McCabe \& Smith, 2002), but not those that precede the test phase (e.g., Anastasi, Rhodes, \& Burns, 2000; finished-PM phase in the present paradigm), have been shown to reduce false recognition. This pattern raises the interesting possibility that the timing of the warning might also affect PM commission errors, though the opposite pattern could emerge (the conjecture being that for PM, the warning at 
encoding would likely not weaken the strong cue-intention encoding, whereas the warning directly preceding test would allow one to try to avoid executing spontaneously retrieved intentions). Differences such as this would indicate that although the retrospective memory literature offers a general guide for theoretical development in the growing PM commission-error literature, potentially dissimilar underlying processes are at play.

Author Notes We thank Allie Plotsky and Sophie Goloff for their assistance. This research was supported by a Psi Chi grant. M.K.S. was partially supported by National Institute on Aging Grants 5T32AG00030 and F32AG041543 and by a Cottrell Fellowship.

\section{References}

Anastasi, J. S., Rhodes, M. G., \& Burns, M. C. (2000). Distinguishing between memory illusions and actual memories using phenomenological measurements and explicit warnings. American Journal of Psychology, 113, 1-26.

Chasteen, A. L., Park, D. C., \& Schwarz, N. (2001). Implementation intentions and facilitation of prospective memory. Psychological Science, 12, 457-461.

Cohen, A.-L., Dixon, R. A., \& Lindsay, D. S. (2005). The intention interference effect and aging: Similar magnitude of effects for young and old adults. Applied Cognitive Psychology, 19, 1177-1197.

Cohen, A.-L., \& Gollwitzer, P. M. (2008). The cost of remembering to remember: Cognitive load and implementation intentions influence ongoing task performance. In M. Kliegl, M. A. McDaniel, \& G. O. Einstein (Eds.), Prospective memory: Cognitive, neuroscience, developmental, and applied perspectives. Mahwah, $\mathrm{NJ}$ : Erlbaum.

Deese, J. (1959). On the prediction of occurrence of particular verbal intrusions in immediate recall. Journal of Experimental Psychology, 58, 17-22. doi:10.1037/h0046671

Einstein, G. O., \& McDaniel, M. A. (1990). Normal aging and prospective memory. Journal of Experimental Psychology: Learning, Memory, and Cognition, 16, 717-726.

Einstein, G. O., McDaniel, M. A., Smith, R. E., \& Shaw, P. (1998). Habitual prospective memory and aging: Remembering intentions and forgetting actions. Psychological Science, 9, 284-288.

Gollwitzer, P. M. (1999). Implementation intentions: Strong effects of simple plans. American Psychologist, 54, 493-503.

Jacoby, L. L., Kelley, C. M., \& Dywan, J. (1989). Memory attributions. In H. L. Roediger III \& F. I. M. Craik (Eds.), Varieties of memory and consciousness: Essays in honour of Endel Tulving (pp. 391422). Hillsdale, NJ: Erlbaum.

Johnson, M. K., Hashtroudi, S., \& Lindsay, D. S. (1993). Source monitoring. Psychological Bulletin, 114, 3-28. doi:10.1037/ 0033-2909.114.1.3

Kimmel, S. E., Chen, Z., Price, M., Parker, C. S., Metlay, J. P., Christie, J. D., ... Gross, R. (2007). The influence of patient adherence on anticoagulation control with warfarin: Results from the International Normalized Ratio Adherence and Genetics (IN-RANGE) study. Archives of Internal Medicine, 167, 229-235.

Liu, L. L., \& Park, D. C. (2004). Aging and medical adherence: The use of automatic processes to achieve effortful things. Psychology and Aging, 19, 318-325. doi:10.1037/0882-7974.19.2.318

Marsh, R. L., Hicks, J. L., Cook, G. I., \& Mayhorn, C. B. (2007). Comparing older and younger adults in an event-based prospective memory paradigm containing an output monitoring component. Aging, Neuropsychology, and Cognition, 14, 168-188.

McCabe, D. P., \& Smith, A. D. (2002). The effects of warnings on false memories in young and older adults. Memory \& Cognition, 30, 1065-1077.

McDaniel, M. A., \& Einstein, G. O. (2007). Prospective memory: An overview and synthesis of an emerging field. Thousand Oaks, CA: Sage.

McDaniel, M. A., Einstein, G. O., \& Jacoby, L. L. (2008a). New considerations in aging and memory: The glass may be half full. In F. I. M. Craik \& T. A. Salthouse (Eds.), The handbook of aging and cognition (3rd ed., pp. 251-310). Mahwah, NJ: Erlbaum.

McDaniel, M. A., Howard, D. C., \& Butler, K. M. (2008b). Implementation intentions facilitate prospective memory under high attention demands. Memory \& Cognition, 36, 716-724. doi:10.3758/ MC.36.4.716

McDaniel, M. A., \& Scullin, M. K. (2010). Implementation intention encoding does not automatize prospective memory responding. Memory \& Cognition, 38, 221-232. doi:10.3758/MC.38.2.221

McFarland, C., \& Glisky, E. (2012). Implementation intentions and imagery: Individual and combined effects on prospective memory among young adults. Memory \& Cognition, 40, 62-69. doi:10.3758/ s13421-011-0126-8

Meiser, T., \& Rummel, J. (2012). False prospective memory responses as indications of automatic processes in the initiation of delayed intentions. Consciousness and Cognition, 21, 1509-1516. doi:10.1016/ j.concog.2012.05.006

Pink, J. E., \& Dodson, C. S. (2012). Negative prospective memory: Remembering not to perform an action. Psychonomic Bulletin \& Review. doi:10.3758/s13423-012-0337-4

Roediger, H. L., III. (1996a). Memory illusions. Journal of Memory and Language, 35, 76-100.

Roediger, H. L., III. (1996b). Prospective memory and episodic memory. In M. Brandimonte, G. O. Einstein, \& M. A. McDaniel (Eds.), Prospective memory: Theory and application (pp. 149155). Mahwah, NJ: Erlbaum.

Roediger, H. L., III, \& McDermott, K. B. (1995). Creating false memories: Remembering words not presented in lists. Journal of Experimental Psychology: Learning, Memory, and Cognition, 21, 803-814. doi:10.1037/0278-7393.21.4.803

Roediger, H. L., III, Watson, J. M., McDermott, K. B., \& Gallo, D. A. (2001). Factors that determine false recall: A multiple regression analysis. Psychonomic Bulletin \& Review, 8, 385-407. doi:10.3758/ BF03196177

Scullin, M. K., \& Bugg, J. M. (2012). Failing to forget: Prospective memory commission errors can result from spontaneous retrieval and impaired executive control. Journal of Experimental Psychology: Learning, Memory, and Cognition. doi:10.1037/a0029198

Scullin, M. K., Bugg, J. M., \& McDaniel, M. A. (2012). Whoops, I did it again: Commission errors in prospective memory. Psychology and Aging, 27, 46-53. doi:10.1037/a0026112

Scullin, M. K., Bugg, J. M., McDaniel, M. A., \& Einstein, G. O. (2011). Prospective memory and aging: Preserved spontaneous retrieval, but impaired deactivation, in older adults. Memory \& Cognition, 39, 1232-1240. doi:10.3758/s13421-011-0106-z

Scullin, M. K., Einstein, G. O., \& McDaniel, M. A. (2009). Evidence for spontaneous retrieval of suspended but not finished prospective memories. Memory \& Cognition, 37, 425-433. doi:10.3758/MC.37.4.425

Walser, M., Fischer, R., \& Goschke, T. (2012). The failure of deactivating intentions: Aftereffects of completed intentions in the repeated prospective memory cue paradigm. Journal of Experimental Psychology: Learning, Memory, and Cognition, 38, 1030-1044.

West, R., McNerney, M. W., \& Travers, S. (2007). Gone but not forgotten: The effects of cancelled intentions on the neural correlates of prospective memory. International Journal of Psychophysiology, 64, 215-225. 